\title{
Electrical Impedance Tomography Based on Mixed Finite Element Model *
}

\author{
Agah D. Garnadi \\ Department of Mathematics, Institute Pertanian Bogor \\ Jl Raya Pajajaran, Bogor 16144, INDONESIA
}

\begin{abstract}
Electrical Impedance Tomography (EIT) is a non-invasive imaging technology to map the conductivity distribution of material within confined device from electrical measurements made on the device periphery. The reconstruction of the conductivity distribution images is an inverse problem, which is ill-posed, hence is both extremely sensitive to noise and highly computationally intensive. This paper examines an EIT device based on Mixed Finite Element formulation model. The reconstruction algorithms of images based upon a non-linear least squares criteria.
\end{abstract}

Keywords: Electrical Impedance Tomography, III-posed problem, Mixed Finite Element Method

*Proceedings CMSE'97, Bandung 1997. [IV.C.6-7] 


\section{Intoduction}

Electrical Impedance Tomography (EIT) is a non-invasive imaging technology to map the conductivity distribution of materials within confined device from electrical measurements made on the device periphery. Based on the measurement data, a reconstruction algorithms is used to reveal the conductivity distribution in the forms of images. The reconstruction of the conductivity distribution images is an inverse problem, which is ill-posed, hence is both extremely sensitive to noise and highly computationally intensive.

The range of applications of EIT as a non-invasive imaging techniques is quite wide. In the biomedical applications, this technology used as a monitoring device, which is non-ionizing, and has large clinical applications ${ }^{(8)}$. Also within geoscience applications, EIT proved to be an effective method as a noninvasive device for subsurface environmental monitoring ${ }^{(9)}$. Even though it is proven as a promising device and cost effective, however to produce an image represents the conductivity distribution from collected data through a reliable and robust image reconstruction algorithms is a challenge.

The electrical fields governing the EIT device is discretized using mixed finite element formulation is the presence of potential fields and electrical current as a set of primary variables. Many formulations for EIT based on direct finite element methods of electrical fields ${ }^{(4)}$, i.e. inherently only deals with potential field's variable. However, in actual measurement at the periphery, there are two variables involved, electrical potential and injected electrical current. Most of the algorithms handles the electrical current as processed variables, not treated as primary variables ${ }^{(4,7,15)}$. In this paper we restrict our study to two-dimensional model which can be represents, for examples, a lateral slice of human thorax or cylindrical process vessels.

Based on the proposed finite element model, we approximate the conductivity distribution of the materials using piece-wise basis function, in our case is Ravi art-Thomas basis, implicitly through the electrical field's approximation. The reconstruction algorithms of images from data collected through boundary measurement is formulated as a nonlinear least-squares problem. The problem solved using Gauss-newton minimization algorithms. To suppress the ill-conditioning inherently present in the approximate Hessian needed in the Gauss-Newton solution, a particular numerical method is proposed.

\section{The forward problem}

The field's equation that govern the electrical impedance measurement are:

$$
\begin{aligned}
\nabla \cdot \sigma \nabla u & =0, \text { in } \Omega \\
u & =\mathcal{U}_{1} \text { on } e_{\ell}, \ell \in\left[1, \ldots, n_{e}\right]
\end{aligned}
$$




$$
\sigma \frac{\partial u}{\partial n}=0, \text { on } \partial \Omega \backslash \cup_{l=1}^{n e} e_{\ell}
$$

Where $\sigma$ is the conductivity distribution, which is everywhere real and positive, $u$ is the potential distribution, $e_{l}, l-t h$ electrode position, $U_{l}$ Voltages measurement at $l-t h$ electrode position, $n$ the outward normal, $\Omega$ the domain of interest, and $\partial \Omega$ its boundary. We restrict ourselves to $\Omega$ a two dimensional bounded domain, the discussion is easily extended to three-D in relatively simple domain and conductivity distributions, however for general problem which is not simple, a numerical methods is used to obtains an approximate solution. In our case, a mixed finite element methods is taken as such a tool.

By introducing new variable $p=-\sigma \nabla u$. Let us rewrite above problem as follow:

$$
\begin{aligned}
\nabla u & =-\sigma^{-1} p, \text { in } \Omega \\
\nabla . p & =0, \text { in } \Omega \\
u & =\mathcal{U}_{\ell} \text { on } e_{\ell} \in\left[1, \ldots, n_{e}\right] \\
p . n & =0, \text { on } \partial \Omega \backslash \cup_{l=1}^{n e} e_{l}
\end{aligned}
$$

Our FEM formation is approximating the above coupled equation in the following manner. First the domain $\Omega$ is partitioned into a number of elements using triangulation $\mathcal{T}_{h}=\cup T_{k}$. For our basis function, we choose the lowest order Raviart-Thomas basis over triangular element [3]. After an assembly element-by-element, the approximating statement can be stated as:

where

$$
\left(\begin{array}{cc}
-D & A \\
A^{T} & 0
\end{array}\right)\left(\begin{array}{l}
p \\
u
\end{array}\right)=\left(\begin{array}{l}
b \\
0
\end{array}\right)
$$

where:

$$
\begin{gathered}
d_{i j}=\int_{\Omega} \sigma^{-1} \psi_{i} \psi_{j} d \Omega ; i, j=1, \ldots, n E \\
a_{i j}=\int_{\Omega} \operatorname{div}\left(\psi_{i}\right) \phi_{j} d \Omega ; i,=1, \ldots, n E: j=1, \ldots, n E
\end{gathered}
$$

The block matrix in the lefthandside is non-singular, while the column vector $\mathrm{C}$ is non-zero due to the presence of a non-zero Dirichlet boundary datum at the injection current electrode nodes.

\section{The Reconstruction Algorithm}

The mixed FEM formulation gives us an approximation of potential within domain of interest for given conductivity distribution and appropriate boundary data. EIT expects to solve the inverse problem: given the boundary conditions and potential reads out at the boundary, solve for the conductivity distribution. The hardest point in EIT is that the boundary potential depends on the conductivity at every point in a nonlinear way.

To solve this problem define: 
1. $v_{m}$ The measured voltage at the boundary.

2. $f$ The boundary voltage obtained by solving the finite element model.

3. g a conductivity distribution with $\mathrm{m}$ degrees of freedom.

4. $\phi=\frac{1}{2}\left(f-v_{m}\right)^{T}\left(f-v_{m}\right)$ The squared error of the reconstruction.

Our problem in impedance imaging is finding $g^{*}$ that minimizing the objective function. To solve this nonlinear problem, we use the Gauss-Newton method. At k-th iteration with estimate $g^{k}$, the estimate value of $g^{*}$ is updated to.

where

$$
\begin{gathered}
g^{*(k+1)}=g^{* k}+\Delta g^{* k} \\
\Delta g^{* k}=-\left[\left[f^{\prime}\right]^{T}\left[f^{\prime}\right]\right]^{T}\left(f-v_{m}\right)
\end{gathered}
$$

And $f^{\prime}$ is total derivative of $\mathrm{f}$ with respect to $\mathrm{g}$, evaluated at $g^{* k}$. The iterative procedure is stopped when the estimate value $g^{*}$ produces small value of $\phi$ within predetermined tolerance.

If we measured at least $m$ linearly independent voltage, the Jacobean matrix $f^{\prime}$ will be of rank $m$. Hence the approximate Hessian, $\left[\left[f^{\prime}\right]^{T}\left[f^{\prime}\right]\right]$, will be of full rank, since it is in a quadratic form, hence it is positive definite. The procedure is guaranteed to converge due to this positive definiteness facts

It is quite likely that the Hessian matrix, $\left[\left[f^{\prime}\right]^{T}\left[f^{\prime}\right]\right]$ is ill-conditioned. To overcome this ill-conditioning we use the marquardt method.

\section{Calculation of the Jacobian Entry}

The Jacobian entry is calculated element wise via (4) matrix. [15] to calculate the Jacobian, we approximate the resistance:

$\rho=\sigma^{-1}=\Sigma_{k=1}^{n_{T}} a_{k} \hat{\chi}_{k}, \hat{\chi}_{k}=\frac{\chi_{k}}{\text { Area }}$ where $\chi_{k}$, is characteristic function of element $k$, $A r e a_{k}$ denotes the area of $k t h$ element. Using this approximation, the entries of $D$ can be expressed as:

$$
d_{i j}=\int_{n} \sum_{k=1}^{n_{T}} a_{k} \hat{\chi}_{k} \psi_{i} \psi_{j} d \Omega=\sum_{k=1}^{n_{T}} a_{k} \int \chi_{k} \psi_{i} \psi_{j} d \Omega
$$

Differentiating (4) with respect to $a_{m}$,

$$
\frac{\partial}{\partial a_{m}}\left(\begin{array}{cc}
-D & A \\
A^{T} & 0
\end{array}\right)\left(\begin{array}{l}
p \\
u
\end{array}\right)+\left(\begin{array}{cc}
-D & A \\
A^{T} & 0
\end{array}\right) \frac{\partial}{\partial a_{m}}\left(\begin{array}{l}
p \\
u
\end{array}\right)=0
$$


Hence:

$$
\frac{\partial}{\partial a_{m}}\left(\begin{array}{l}
p \\
u
\end{array}\right)=\left(\begin{array}{cc}
-D & A \\
A^{T} & 0
\end{array}\right)^{-1} \frac{\partial}{\partial a_{m}}+\begin{array}{cc}
-D & A \\
A^{T} & 0
\end{array}\left(\begin{array}{l}
p \\
u
\end{array}\right)
$$

Calculating the derivatives on the right hand side:

$$
\frac{\partial}{\partial a_{m}}\left(\begin{array}{l}
p \\
u
\end{array}\right)=-\left(\begin{array}{rr}
-D & A \\
A^{T} & 0
\end{array}\right)^{-1}\left(\begin{array}{rr}
-D & 0 \\
0 & 0
\end{array}\right)^{-1}\left(\begin{array}{c}
P \\
u
\end{array}\right)
$$

where:

$$
d_{i j}=\int_{\Omega} \hat{\chi}_{m} \psi_{i} \psi_{j} d \Omega
$$

Hence:

$$
\frac{\partial}{\partial a_{m}}\left(\begin{array}{l}
p \\
u
\end{array}\right)=-\left(\begin{array}{rr}
-D & A \\
A^{T} & 0
\end{array}\right)^{-1}\left(\begin{array}{rr}
-D & 0 \\
0 & 0
\end{array}\right)\left(\begin{array}{rr}
-D & A \\
A^{T} & 0
\end{array}\right)^{-1}\left(\begin{array}{l}
b \\
0
\end{array}\right)
$$

\section{Result}

We simulate a two-dimensional unit radius inhomogeneous conductivity distribution embedded in a unit cube. We used 16-electrodes equally space on the cube boundary. Hence we were able to simulate $\mathrm{n}=104$ independent measurements

Our test is a problem with piecewise constant resistivity representation. This is a standard test in the geophysics and medicine area. We use the maximal contrast ratio 4.5:1 for the resistivity distribution. The object to be reconstructed is a rectangular object of $350 \Omega \mathrm{cm}$ imbedded in a $75 \Omega \mathrm{cm}$.

A complete results will be presented in poster session at this conference

\section{Acknowledgment}

I would like to thanks the Commonwealth Government of Australia, c/q Ausaid (aka AIDAB) that made me possible to study at the department of mathematics, schools of mathematical sciences, The Australian National University for 1991-1993 financial years. I'd like to thanks ICTP for funding my stays in Trieste on two occasions in September 1996 and May-June 1997 periods 


\section{References}

[1] M. Cheney and Isaacson, 1995, Issues in Electrical Impedance Imaging ,IEEE Computational science and Engineering Magazine, v2(4), 53-62.

[2] K. A. Dines and R. J. Little, 1981, Analysis of electrical conductivity imaging, geophysics, v 46, 1025-1036.

[3] R. S. Falk and E. J. Osborn, 1994, Remarks on Mixed Finite Element Methods for Problem with Rough Coefficients, Math, Comp, v 62, 1-19.

[4] P. Hua and E. J. Woo, 1990, Reconstruction Algorithms, in [13], 97-137.

[5] D. Isaacson, M. Cheney, and J. Newell, 1992, Problem in Impedance Imaging in L. Paivarinta and E. Somersault (ad), Proceeding of the Lapland Conference on Inverse Problem, pp 62-70.

[6]C. Johnson, 1990, Numerical Solution of Partial Differential Equations by the Finite Element method, Cambridge university Press.

[7] T. Murai and Y. Kagawa, 1985, Electrical Impedance Tomography Based on a Finite Element Model, IEEE Trans. Biomed. Eng., v BME-32, 177-184.

[8] J. C. Newell, 1996, State of the art in Impedance Imaging, Lecture Notes distributed at College on medical Physics: Methods, Instrumentation and Techniques in Medical Imaging, 9-27 September 1996, ICTP, Trieste.

[9] F. Santosa, 1994, Inverse problem holds key to safe, continuous imaging, SIAM News, vol 27(6), Pl. 
[10] G. Strang, 1988, A framework for equilibrium equations, SIAM Rev, 30, 283-297.

[11] S. A. Vavasis, 1994, Stable numerical algorithms for equilibrium systems, SIAM J. Matrix Anal. Appl, v 15, 1108-1132.

[12] S. A. Vavasis, 1996, Stable finite elements for problems with wild coefficients, SIAM J. Numer. Anal., v 33 (3), 890 -916.

[13] J. G. Webster, 1990, Electrical Impedance Tomography, Adam Hilger.

[14] J. G. Webster, 1990, Electrical Impedance Tomography, in [13], 1-7.

[15] T. J. Yorkey, 1990, Electrical Impedance Tomography with piecewise polynominal conductivities, J. Comp. Phys., 91 344-360. 